https://helda.helsinki.fi

Fluid management in acute kidney injury

Perner, Anders

2017-06

Perner , A , Prowle , J , Joannidis , M , Young , P , Hjortrup , P B \& Pettilä , V 2017 , ' Fluid management in acute kidney injury ' , Intensive Care Medicine , vol. 43 , no. 6 , pp. 807-815 . https://doi.org/10.1007/s00134-017-4817-x

http://hdl.handle.net/10138/237046

https://doi.org/10.1007/s00134-017-4817-x

unspecified

publishedVersion

Downloaded from Helda, University of Helsinki institutional repository.

This is an electronic reprint of the original article.

This reprint may differ from the original in pagination and typographic detail.

Please cite the original version. 


\title{
REVIEW
}

\section{Fluid management in acute kidney injury}

\author{
Anders Perner ${ }^{1 *}$ D, John Prowle ${ }^{2}$, Michael Joannidis ${ }^{3}$, Paul Young ${ }^{4}$, Peter B. Hjortrup ${ }^{1}$ and Ville Pettilä5
}

(C) 2017 Springer-Verlag Berlin Heidelberg and ESICM

\begin{abstract}
Acute kidney injury (AKI) and fluids are closely linked through oliguria, which is a marker of the former and a trigger for administration of the latter. Recent progress in this field has challenged the physiological and clinical rational of using oliguria as a trigger for the administration of fluid and brought attention to the delicate balance between benefits and harms of different aspects of fluid management in critically ill patients, in particular those with AKI. This narrative review addresses various aspects of fluid management in AKI outlining physiological aspects, the effects of crystalloids and colloids on kidney function and the effect of various resuscitation and de-resuscitation strategies on the course and outcome of AKI.
\end{abstract}

Keywords: Acute kidney injury, Critical Care, Fluid, Intravenous fluid, Kidney failure, Renal failure, Sepsis, Shock

\section{Introduction}

Hypovolaemia is accepted as a major risk factor for the development of acute kidney injury (AKI) [1] and is associated with low urinary output [2]. Moreover, oliguria often occurs as the first clinical sign of AKI [3] and is one of the two criteria defining AKI according to the KDIGO guidelines [4]. Hence, it is understandable that oliguria was the second most frequent trigger for fluid administration in critically ill patients in the international FENICE point prevalence study [5]. Considering that in the critically ill the two most frequent aetiologies of AKI are sepsis and hypovolaemia [6], timely fluid administration may be a preventive measure against AKI and should be effective both through restoration of circulating volume and improving impaired renal perfusion. However, there are many uncertainties about the benefit of fluid administration for the prevention and treatment of AKI. Many forms of AKI are considered volume unresponsive [1], in particular in cases that are not hypovolaemic and if AKI is not caused by renal hypoperfusion, but rather nephrotoxics or

\footnotetext{
*Correspondence: anders.perner@regionh.dk

${ }^{1}$ Department of Intensive Care, Rigshospitalet, University of Copenhagen, Copenhagen, Denmark

Full author information is available at the end of the article
}

renal inflammation. In those situations injudicious use of fluids carries its own risks of contributing to the development or worsening of AKI by fluid overload (Fig. 1) and sometimes necessitating initiation of renal replacement therapy (RRT) [7]. Fluid overload may even impair renal recovery after AKI [8]. Overall the risk-benefit balance of fluid administration in AKI depends on the aetiology of AKI, the volume status of the patients, the types of fluid used and likely also the timing, rates and volumes used. This review addresses various aspects of fluid management in AKI specifying the physiological rationing and the effects of crystalloids and colloids on kidney function and outlining the effects of various resuscitation and de-resuscitation strategies on the course and outcome of AKI.

\section{Physiological rationale for fluid management in AKI}

The physiological rationale for administration of fluids in critically ill patients is to restore tissue perfusion. In absolute hypovolaemia, renal perfusion may be compromised as a result of decreased cardiac output (CO). Thus, in that case fluid therapy seems a logical option to increase the stroke volume (SV) and CO, renal blood flow (RBF), renal oxygen supply, and glomerular filtration rate (GFR). However, AKI or oliguria per se may not reflect renal

\section{实




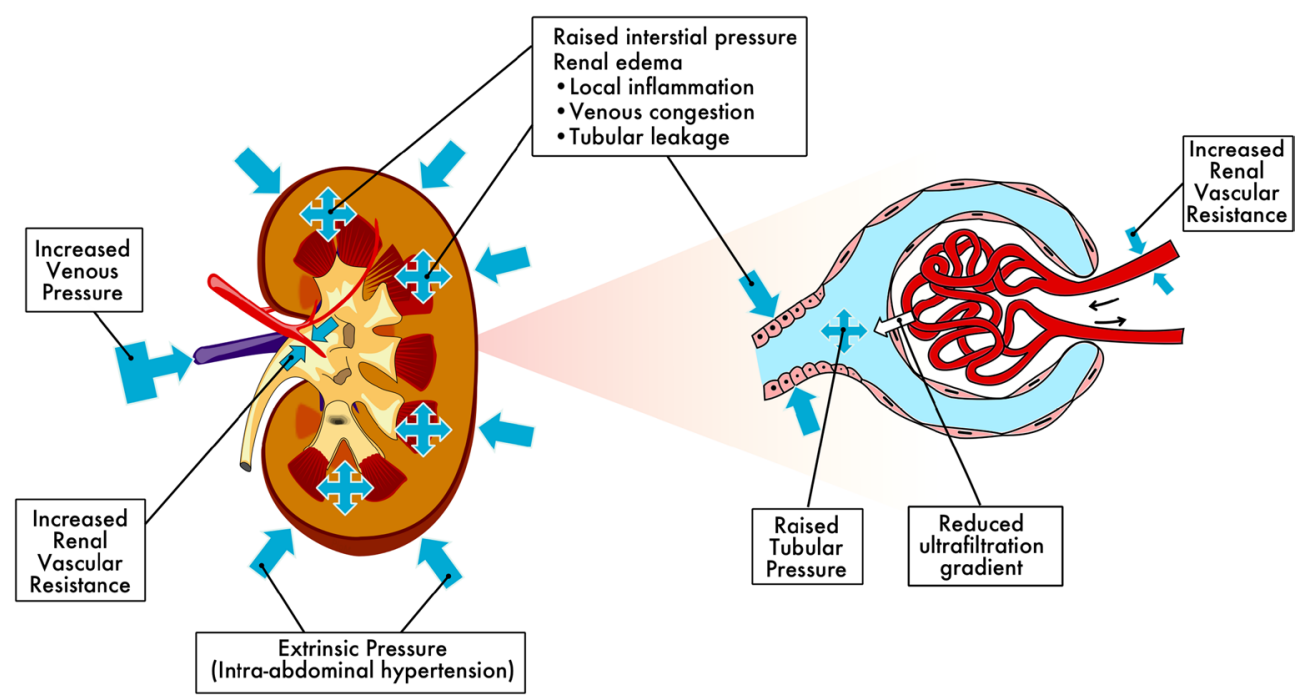

Fig. 1 Fluid overload and interstitial oedema can contribute to the maintenance of AKI. Increased central venous pressure reduces the transrenal pressure gradient for RBF, while increased interstitial and tubular pressure may reduce or abolish the net glomerular filtration pressure gradient. Increased preglomerular resistance, in response to tubular injury, further reduces RBF and glomerular capillary hydrostatic pressure and hyperchloraemia might contribute to this effect, which represents a pathophysiological activation of the tubulo-glomerular feedback mechanism. Finally, development of intra-abdominal hypertension restricts venous drainage and extrinsically compresses the kidney [64]. AKI acute kidney injury, GFR glomerular filtration rate, $R B F$ renal blood flow

macrovascular hypoperfusion. Additionally, only severe oliguria is associated with development of creatininebased AKI $[9,10]$. Of note, fluid administration causes increased workload to kidneys because of increased filtration of sodium chloride leading to increased reabsorptive activity, and increased consumption of $\mathrm{O}_{2}$ and ATP in tubular cells.

In addition to RBF, colloid osmotic pressure of plasma proteins and glomerular arterial tone are factors that affect the pressure gradient between glomerular capillary and Bowman's space (Fig. 1). GFR is dependent on this pressure gradient. Thus, increasing $\mathrm{CO}$ may lead to increased RBF and increased GFR. However, in established AKI, RBF and GFR seem to correlate poorly [11]. Furthermore, in early experimental septic AKI, RBF is often normal or even higher [12]. Thus, fluids aimed to increase RBF may not have the desired effect on GFR if $\mathrm{CO}$ is normal or increased. The RBF fraction of CO may also be reduced from normal in septic patients [13]. Intent to maintain or increase renal oxygen delivery by administration of fluids may also be questioned, because in AKI the metabolic activity is decreased with decreasing GFR, although both animal [14] and clinical data [15] suggest that sodium reabsorption becomes less metabolically efficient in AKI. Moreover, the causal relationship between periodic ischaemia and development of new AKI has been rejected [16].
In clinical practice, indications for fluid therapy other than absolute hypovolaemia are unclear. Changes of RBF, renal oxygen supply or GFR are not measured in clinical practice. However, fluids are often administered to critically ill patients on the basis of other indications for prevention or treatment of AKI, although no generally accepted rules regarding indications, timing, choice of fluid, rates and volumes, or duration of fluid therapy exist.

While hypotension (59\%) and oliguria (18\%) are the most frequent indications for fluid administration in ICU patients [5], there are limited physiological rationale and clinical data to support the benefits of fluids in these situations. Activation of the renin-angiotensin system and increased antidiuretic hormone may cause retention of water and salt, which may be further aggravated by excess fluid therapy. In septic shock, the primary pathophysiologic phenomena are arterial and venous dilatation, causing a vasoplegic state not restored by giving fluids but rather vasoconstricting agents to alleviate hypotension. Additionally, microvascular thrombosis, endothelial injury and shedding of glycocalyx lead to abnormal microcirculation [17] and increased capillary leak [18] decreasing the potential benefits of fluids. However, the promotion of diuresis in oliguric states by fluid therapy may seem logical in the prevention of rhabdomyolysis and IV-contrast-induced AKI, but may not be beneficial in the latter cases [19]. The advantages of fluids in 
oliguric states related to acute illness are less clear. There seems to be dissociation between macrohaemodynamic response to fluid challenge and renal response; half of the oliguric ICU patients are not renal responders [20]. Therefore, the use of hypotension or oliguria alone as triggers for fluid therapy is often not fully supported by physiological reasoning. Notably, haemodilution caused by excess fluids may be deleterious. In support of this notion, animal data suggest that $\mathrm{RBC}$ transfusion may improve renal microvascular oxygenation [21], but confirmatory human data have been inconclusive.

Of note, the effects of fluid therapy are plausibly highly dependent on the phase of acute illness conceptualized into four different phases: rescue, optimization, stabilization and de-escalation [22]. Early beneficial effects of fluids in the resuscitation phase may turn to deleterious fluid accumulation in later phases. Similarly, triggers such as oliguria may indicate hypovolaemia and decreased tissue perfusion in the early resuscitation phase but may indicate established AKI [4] later in the course of critical illness.

The impacts of choice of fluid, volume, rate and duration of administration of fluids are discussed in the following paragraphs.

\section{Colloid solutions}

It has been a common belief that the administration of colloid solutions to critically ill patients would reduce the overall need for fluid as compared with the administration of crystalloids. We may estimate the potential fluidsparing effects of colloid use vs. crystalloid use using data from the recent blinded randomised trials. These data indicate a modest fluid-sparing effect of colloids at least in general ICU patients (Table 1). In patients with sepsis, this effect may be limited.

In the last decade there have been major changes in the use of IV fluids in critical care and ICU settings, in particular that of the colloid solutions [23]. The changes in fluid practice occurred after the publication of large trials

Table 1 Colloid-to-crystalloid volume ratios of the masked fluid administration in the four randomised, blinded trials of ICU patients $[24,26,35,77]$

\begin{tabular}{llclll} 
Trial & Patients & Patient no. & Colloid & Crystalloid & Ratio \\
SAFE & ICU & 6997 & $\begin{array}{c}\text { Albumin } \\
4 \%\end{array}$ & Saline & $1.0: 1.4$ \\
Crystmas & $\begin{array}{c}\text { Sepsis in } \\
\text { ICU }\end{array}$ & 196 & HES 6\% & Saline & $1.0: 1.1$ \\
6S & $\begin{array}{c}\text { Sepsis in } \\
\text { ICU }\end{array}$ & 798 & HES 6\% & Ringer's & $1.0: 1.1$ \\
Chest & ICU & 7000 & HES 6\% & Saline & $1.0: 1.2$ \\
\hline
\end{tabular}

HES hydroxyethyl starch and updated systematic reviews showing increased rates of AKI and use of RRT with hydroxyethyl starch (HES) in critically ill patients $[24,25]$, including those with sepsis [26-28] and increased mortality in patients with sepsis $[26,27]$. As a result, the Surviving Sepsis Campaign (SSC) guideline recommended against the use of HES [29], the FDA issued a boxed warning for HES based on the risk of AKI [30], and the European Commission made the legally binding decision that HES can no longer be used in critically ill patients including those with sepsis and burn injury [31].

Gelatine is the other widely used synthetic colloid solution; however, there are very limited data on the benefits and harms, including risk of kidney impairment of this colloid. A recently updated systematic review included only three trials assessing rates of AKI in only 212 patients randomised to gelatine vs. crystalloid/albumin; the point estimate indicated a $35 \%$ increased relative risk of AKI with gelatine [32]. Even though this result was not statistically significant it supports the associations of increased risk of AKI with gelatine use in before-andafter cohort studies done in patients with sepsis [33] and those undergoing cardiac surgery [34].

Albumin is a natural colloid and appears to be safe to use in patients at risk or with established AKI. Thus the requirements for RRT were similar in the albumin and saline groups of the SAFE trial, in which 6997 ICU patients with clinical signs of hypovolaemia were randomised [35]. Similar effects were observed in the randomised open-label ALBIOS trial, in which 1818 patients with severe sepsis or septic shock received $60 \mathrm{~g}$ albumin per day targeting a serum albumin level of greater than $30 \mathrm{~g} / \mathrm{L}$ vs. no albumin; neither rates of AKI nor use of RRT differed between the groups [36]. These results are supported by the recent network meta-analysis finding no difference in the use of RRT with albumin vs. crystalloid solutions in patients with sepsis with moderate certainty [28]. In both the SAFE and ALBIOS trials, net fluid balances were less positive in the albumin vs. control groups, but the differences were modest. As no outcomes differed with statistical significance between the intervention groups in these trials, the potential benefit of less positive fluid balance with the use of albumin remains to be documented.

Should colloid solutions be used in patients with AKI or those at risk of AKI? Within the present evidence base, we can recommend with high certainty that HES should not be used in these patients (Fig. 2). For the use of gelatines a similar recommendation appears warranted for patients with AKI even though the certainty is much lower for the reasons given above (Fig. 2). Albumin, on the other hand, appears to be safe in these patients, but at the same time there appears to be limited benefit from 


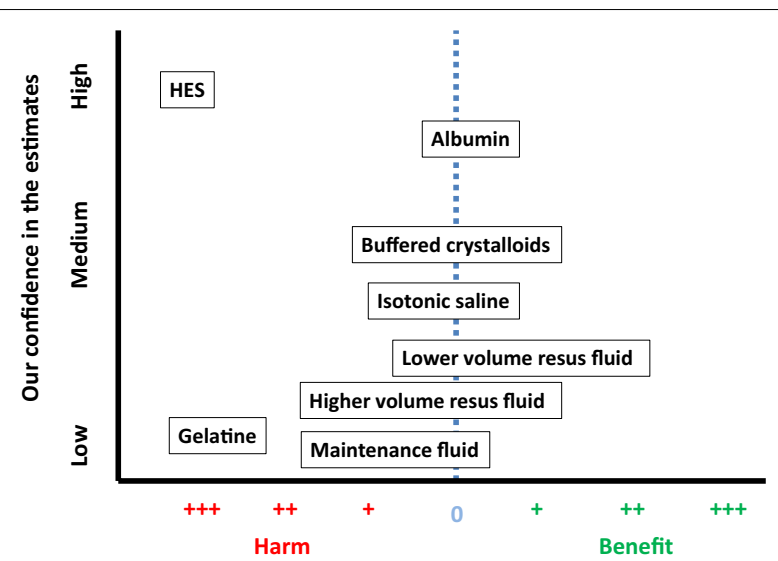

Fig. 2 Summary of the estimated benefits and harms regarding kidney function of the fluid interventions covered in this review. Zero on the $x$-axis indicates neutral effect; deviations of the text boxes from zero indicate estimated benefit or harm. We have given our confidence in the estimates on the $y$-axis. HES hydroxyethyl starch

albumin as compared to crystalloid solutions [28, 37] (Fig. 2). As the use of albumin may be on the rise in ICUs [23], we need large trials assessing which settings, protocols or subgroups of critically ill patients may benefit from this expensive and limited resource.

\section{Crystalloid solutions}

While crystalloids are now the accepted first-line IV fluid in most ICU patients, the most appropriate crystalloid to use in patients with AKI is unclear. The impact of the chloride composition of crystalloid fluids on renal function has been the focus of research because in animal models increasing plasma chloride levels produce progressive renal vasoconstriction and a GFR [38]. This effect is demonstrable in healthy volunteers where renal artery blood flow velocity and renal cortical tissue perfusion fall after administration of $2 \mathrm{~L}$ of $0.9 \%$ saline, which has a higher chloride composition than normal plasma, but not after administration of $2 \mathrm{~L}$ of a buffered crystalloid with a similar chloride concentration to plasma [39]. Similar effects have been shown in a number of animal experiments [40,41]. Although these observations lend plausibility to the hypothesis that the chloride composition of IV fluids can alter their effect on GFR, it is unclear whether use of $0.9 \%$ saline increases the risk of development or progression of renal dysfunction. In particular, because oxygen is offloaded to the tissues more effectively as pH falls (as a result of the Bohr effect [42]) and chloride-rich solutions are acidifying [43], it is unclear whether $0.9 \%$ saline impairs or enhances renal tissue oxygen delivery compared to buffered crystalloids. There are no published clinical trials specifically comparing different crystalloids in the setting of AKI and although a recent meta-analysis suggested that the use of high chloride fluids was associated with a $60 \%$ increase in the risk of developing acute kidney injury [44], the statistical significance of this observation was dependent on the findings of a single before-and-after study [45]. In this before-and-after study [45] chloride-rich fluids, including a potentially nephrotoxic gelatin-based colloid [33, 46], were removed from the ICU following a period of observation, and the influence of unmeasured confounders may have contributed to fluctuations in AKI incidence over time [47].

Two observational studies not included in the aforementioned meta-analysis [44] reported no significant association between choice of intravenous crystalloid and AKI risk [48, 49]. In addition, a recent network metaanalysis comprising 14 randomised controlled trials (RCTs) in septic patients showed that the use of buffered crystalloids compared with saline was not associated with a difference in RRT requirements [28]. In the Saline vs. Plasma-Lyte $148^{\circledR}$ (a gluconate/acetate-buffered crystalloid) for ICU fluid Therapy (SPLIT) trial there were no significant between-group differences in serum creatinine levels, rates of AKI, or requirements for RRT [50]. Similarly, in the recent Balanced Crystalloids vs. Saline in the ICU trial [51] (the SALT trial) there were no between-group difference in serum urea or creatinine measures, major adverse kidney events (in-hospital mortality, receipt of new RRT or final inpatient serum creatinine of at least $200 \%$ of baseline) recorded up until 30 days post enrolment.

In the SPLIT trial [50] and the SALT trial [51] the volumes of crystalloid delivered to patients were small (median of $2 \mathrm{~L}$ and $1.5 \mathrm{~L}$, respectively) and both study populations were dominated by low acuity patients. As a result, it is plausible that the dose of $0.9 \%$ saline administered in these studies was insufficient to cause clinically evident renal toxicity even if the potential for such toxicity exists. In the SALT study, among patients who received the largest volumes of crystalloid, there appeared to be more AKI in patients who received 0.9\% saline [51]. However, systematic differences between patients that received high volumes of $0.9 \%$ saline compared with patients that received high volumes of buffered crystalloids cannot be excluded. As a result, the comparison is subject to bias and may not reflect a causal relationship between $0.9 \%$ saline use and AKI risk. In a recent cohort study of ICU patients who received large volume fluid resuscitation, defined as greater than $60 \mathrm{~mL} / \mathrm{kg}$ over a 24-h period, there was no robust association between chloride load and AKI risk after adjusting for illness severity [52].

Low-quality data raise the possibility that buffered crystalloids may be associated with a lower AKI risk than 
$0.9 \%$ saline in some settings, and preliminary data suggest that the buffered crystalloids lactated Ringer's and Plasma-Lyte $148^{\circledR}$ can be used safely in the critically ill $[50,51]$. There are no data comparing different buffered crystalloids in patients with AKI or evaluating whether or not the choice of buffered crystalloid affects the risk of AKI developing. For now, at least it appears that when it comes to the risk of development or progression of AKI, $0.9 \%$ saline and buffered crystalloids are all acceptable choices for IV fluid management in critically ill patients (Fig. 2).

\section{Fluid volumes}

One of the goals in the landmark trial of Early GoalDirected Therapy (EGDT) in septic shock by Rivers and colleagues was a urinary output of at least $0.5 \mathrm{~mL} / \mathrm{kg} / \mathrm{h}$, but the protocol did not specify how to achieve this goal [53]. As stated above, absolute hypovolaemia causes oliguria, which may have led to the notion that low urine output may be due to decreased renal perfusion in critically ill and that fluid administration will alleviate the condition. This is likely to be oversimplified-especially in the case of septic AKI [54]. Also, a potential harmful effect of increased fluid balance has been suggested by observational data indicating increased risk of AKI with increasing central venous pressure (CVP) in adjusted analyses of ICU patients [55]. Nevertheless, low urine output is still one of the most frequent indications for fluid administration in critically ill patients [5]. Similarly, a study in severe sepsis and septic shock found oliguria as an indication for a fluid bolus in $26 \%$ of cases, but interestingly the urinary output remained unchanged $1 \mathrm{~h}$ following a fluid bolus [56]. In a worldwide survey of intensive care specialists, almost half of the respondents expected an increase in urinary output of more than $20 \mathrm{~mL} / \mathrm{h}$ in order to constitute a positive response to fluid administration [57]. Recent data from the randomised CLASSIC trial suggest that additional fluid may not increase urinary output [58] (Fig. 3). Thus, there may be dissociation between the expectation of clinicians to the response of a fluid bolus in terms of increased urinary output and the observed response. In the case of a modest response in urinary output to a 1-L fluid administration (e.g. a $5 \mathrm{~mL} / \mathrm{h}$ increase), the extra fluid would take days to excrete without other interventions.

Although urine output is a frequent indication for fluid administration, there are limited data to support this practice, and high-quality data on fluid volumes and AKI from RCTs are sparse. A systematic review and meta-analysis of RCTs on fluid management in sepsis and ARDS following the resuscitation phase found no statistically significant difference in use of RRT for conservative vs. liberal strategies (risk ratio 0.88; 95\% CI 0.64-1.22),

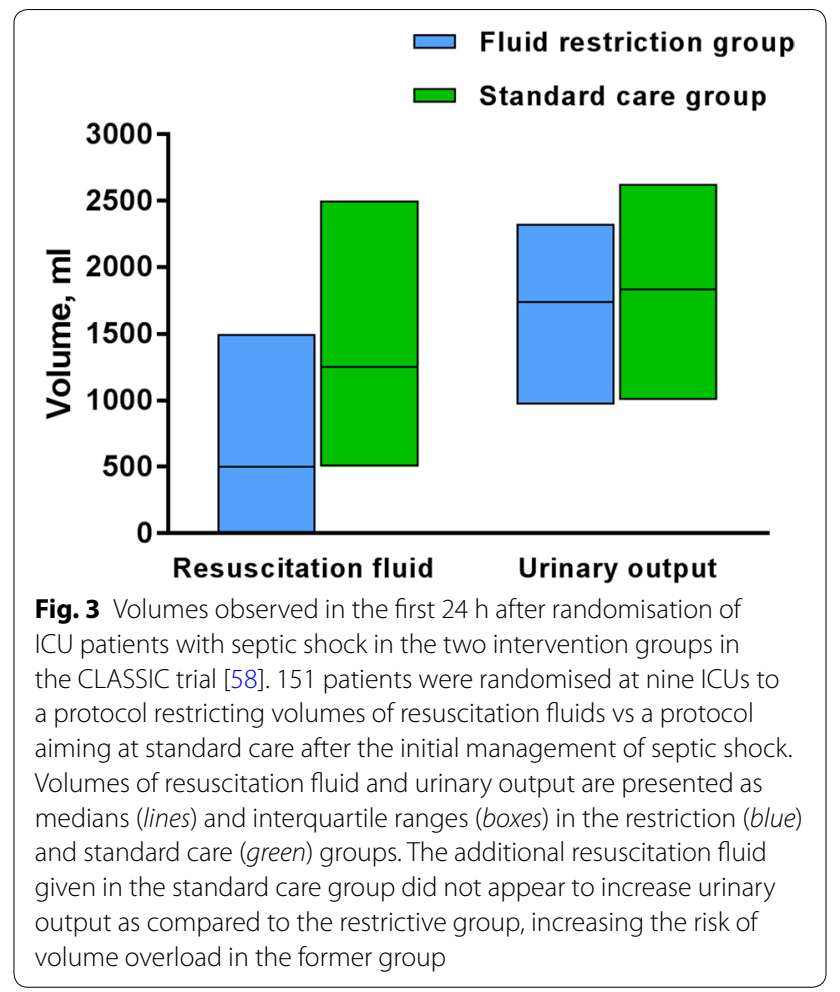

but the analysis was characterized by imprecision as only three trials were included [59]. The CLASSIC trial where patients with septic shock who had received the initial fluid resuscitation were randomised to either a protocol with restrictive fluid resuscitation or a protocol aiming at standard care reported fewer patients with worsening of AKI in the fluid restriction group [58]. In the threearmed PROCESS trial, patients with septic shock randomised to the protocol-based standard therapy group received more fluids and had higher risk of new onset renal failure compared to patients randomised to the EGDT group and the usual care group [60]. Of note, fluids were only one part of the intervention in the trial which also included vasopressors, dobutamine and blood transfusions. Importantly, progression to chronic kidney disease was not an outcome measure in any of these trials; it may be a more robust outcome as it will not be affected by the potential different dilution of creatinine by differences in fluid volumes.

Observational studies have most often assessed the association between mortality and increased fluid balance in AKI rather than specifically fluid input which hampers the interpretation of fluid input, because decreased fluid output as well may lead to increased fluid balance. With this limitation kept in mind, observational studies have indicated harm with increased fluid balance in AKI [61] and in patients receiving renal replacement 
therapy [62]. Even though these analyses were adjusted for illness severity, conclusions must be drawn with caution because of the risk of confounding, and inference about causality cannot be made since the increased fluid balance might be indicative of illness severity not reflected in the summary scores that were used to perform adjustments.

Taken together, there is evidence to suggest that higher fluid inputs may precipitate rather than alleviate AKI, but no firm conclusions can be made from the available data (Fig. 2). Even though higher fluid volumes appear to be associated with harm, the differences in the compared protocols hamper the clinical applicability. AKI comprises a broad spectrum of pathophysiological characteristics, and a 'one size fits all' approach regarding fluid input is unlikely to be obtainable. Nevertheless, a hesitant approach to persistent fluid administration with the aim of increasing urinary output is likely to be prudent (Fig. 2).

\section{De-resuscitation strategies}

Even when fluid resuscitation is carefully guided and early vasopressor support is employed, initial treatment of acute critical illness almost always results in a positive fluid balance and tissue oedema, in particular in patients with AKI. Thus, after this initial phase, treatment focus should shift towards the prevention of further fluid overload and the active removal of accumulated excess salt and water. Adopting such a proactive approach to fluid management involves both appreciation of the balance of fluid inputs and outputs and clinical monitoring for signs of fluid overload [63, 64]. Any strategy to remove fluid must commence with the rational management of fluid input to minimize initial and ongoing fluid accumulation [22] and be accompanied by continuous management of fluid status to prevent fluid overload if obligate intake is in excess of endogenous fluid output and resolving fluid accumulation once stability has been achieved by intervening to increase fluid removal. To achieve fluid removal in excess of spontaneous losses, either to resolve or prevent fluid overload, there are two major options available to the clinician, diuretic pharmacotherapy or extracorporeal ultrafiltration. Choice of diuretic therapy over mechanical fluid removal will be dependent on renal function, baseline urine output, electrolyte status and severity of fluid overload; however, the response to therapy should be regularly reassessed to make sure the choice remains appropriate. While evidence exists that the use of diuretics to treat established AKI is ineffective [65] and may delay definitive AKI management with RRT [66], their use in a large population of patients with AKI in the ICU has not been associated with increased mortality [67]. Thus, the use of diuretics specifically to manage fluid balance in patients may be logical and clinically supportable, as long as response is adequately assessed [68].

When employing an active fluid management strategy with diuretics or mechanical ultrafiltration it is important to distinguish both the overall level of fluid overload (the eventual target) and the capacity to rapidly remove fluid from the circulation without inducing haemodynamic instability. In the sickest patients, extent of fluid overload and tolerance of its removal may be widely disassociated, thereby greatly complicating management of severe fluid overload. Importantly, different forms of monitoring inform clinicians on these differing aspects of therapy; static assessments of fluid status inform on the extent of fluid accumulation, while dynamic assessments of cardiac output and tissue perfusion provide information on tolerance of rate of removal.

Determining the total quantity of fluid overload is challenging as charted fluid balances are often inaccurate and do not account for unmeasured fluid losses nor change in "flesh weight" during prolonged critical illness. However charted daily fluid balance does appear to be a more useful guide in determining risk of fluid overload than daily weight, which can be inaccurate in the critical care setting [69]. However even the most accurate fluid balance will fail to account for uncertain fluid status at ICU admission. Bioelectrical impedance body composition analysis (BIA) is a non-invasive method of fluid assessment which can provide estimates of total body, extracellular and intracellular water to allow the quantification of fluid overload [70]. Similarly, serum N-terminal proB-type natriuretic peptide (BNP) has been examined as a biomarker of cardiac response to circulatory overfilling. In the ICU, fluid overload as defined by BIA and/or BNP has been associated with adverse outcomes; however, these measures are not well correlated [71]. Overall, these methods, while interesting, have not been extensively validated in the critically ill, and their benefit over use of a well-kept fluid balance has not yet been established [72].

During the process of fluid removal physiological assessment of fluid status can be as important as during initial resuscitation, as if fluid removal is excessive or out of pace with vascular refilling hypovolaemia-induced falls in cardiac output can increase the risk of recurrent renal and other organ injury. The commencement of fluid removal can be considered as a "reverse fluid challenge" and demands monitoring in the same fashion as the response to bolus fluid administration. The possibility of other organ injury during fluid removal should be considered both clinically and in the design of studies. Follow-up of a small subgroup of patients from the fluids and catheters treatment trial (FACTT), where conservative fluid management (with diuretics) was associated 


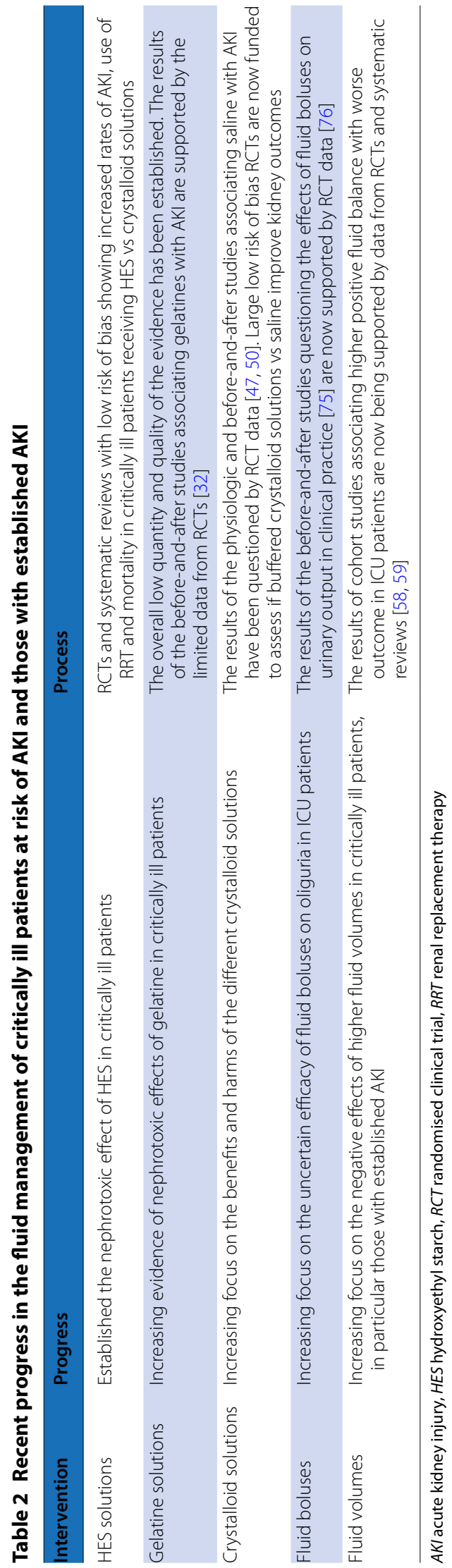

with lesser duration of mechanical ventilation in lung injury, showed poorer cognitive function after recovery from critical illness in patients in the conservative vs. the liberal fluid group. Hypothetically, this could be related to transient episodes of cerebral hypoperfusion during fluid removal [73], as there was a higher incidence of "new shock" in the conservative arm. Recently a simpler "FACCT-LITE" fluid strategy has been described [74]. This approach was associated with similar respiratory and renal outcomes compared to the conservative group in FACCT, but with a "new shock" rate similar to the liberal arm, suggesting the value of haemodynamic stability for fluid removal emphasised in this regimen.

Overall, fluid overload is strongly associated with adverse outcomes in critical illness; however, its resolution can be difficult and prone to complications. Minimizing, as much as possible, the acquisition of fluid overload is thus of key importance. Fluid removal strategies need to be carefully titrated and monitored for haemodynamic tolerance and continuous methods may be better tolerated. Strategies to limit or resolve fluid overload in critically ill adults or children with lung injury or sepsis have been shown to increase the number of ventilator-free days and decrease ICU length of stay; however, the effect on mortality and other long-term outcomes remains uncertain [59]. Large randomised trials considering both short- and long-term clinical outcomes are needed to determine optimal fluid strategies in critically ill patients including those with AKI.

\section{Perspectives}

As detailed above, optimal fluid management has great beneficial potential in critically ill patients at risk of AKI and those with established AKI. On the other hand, there is an imminent risk of harming these patients by the choice, timing, rate and volume of IV fluids. There is an urgent need for better technologies to assess blood volume and hydration status of our patients beyond fluid responsiveness. Bioelectrical impedance vector analysis (BIVA) may hold some promise [72]. We have improved the fluid management of these patients (Table 2) and may continue to do so through the conduct of high-quality clinical research to ensure that we give the right fluid to the right patient at the right time and rate. If so, we will continue to improve the care and overall outcomes of critically ill patients at risk of AKI and those with established AKI.

\footnotetext{
Author details

${ }^{1}$ Department of Intensive Care, Rigshospitalet, University of Copenhagen, Copenhagen, Denmark. ${ }^{2}$ Adult Critical Care Unit, The Royal London Hospital, Barts Health NHS Trust, William Harvey Research Institute, Queen Mary University of London, London, UK. ${ }^{3}$ Division of Intensive Care and Emergency Medicine, Medical University Innsbruck, Innsbruck, Austria. ${ }^{4}$ Intensive Care Unit, Medical Research Institute of New Zealand, Wellington Hospital, Wellington,
} 
New Zealand. ${ }^{5}$ Division of Intensive Care Medicine, Department of Anesthesiology, Intensive Care and Pain Medicine, University of Helsinki and Helsinki University Hospital, Helsinki, Finland.

\section{Compliance with ethical standards}

\section{Conflicts of interest}

AP is member of the steering committee and national investigator of a vasopressin trial in septic shock sponsored by Ferring Pharmaceuticals; his department is reimbursed for his time. The department also receives research funding from Fresenius Kabi and CSL Behring. MJ is a consultant or speaker for Baxter, Fresenius, Asahi Kasei, Astute, CSL Behring. PY is a member of the Plasmalyte vs. Saline (PLUS) trial management committee. Baxter Healthcare is providing fluids for this trial.

Received: 14 February 2017 Accepted: 22 April 2017

Published online: 3 May 2017

\section{References}

1. Himmelfarb J, Joannidis M, Molitoris B et al (2008) Evaluation and initial management of acute kidney injury. Clin J Am Soc Nephrol 3:962-967. doi:10.2215/CJN.04971107

2. Lehner GF, Forni LG, Joannidis M (2016) Oliguria and biomarkers of acute kidney injury: star struck lovers or strangers in the night? Nephron 134:183-190. doi:10.1159/000447979

3. Ostermann M, Joannidis M (2016) Acute kidney injury 2016: diagnosis and diagnostic workup. Crit Care 20:299. doi:10.1186/ s13054-016-1478-z

4. Kidney Disease: Improving Global Outcomes (KDIGO) Acute Kidney Injury Work Group (2012) KDIGO clinical practice guideline for acute kidney injury. http://www.kdigo.org/clinical_practice_guidelines/pdf/KDIGO\%20 AKI\%20Guideline.pdf. Accessed 1 Jan 2017.

5. Cecconi M, Hofer C, Teboul J-L et al (2015) Fluid challenges in intensive care: the FENICE study: a global inception cohort study. Intensive Care Med 41:1529-1537. doi:10.1007/s00134-015-3850-x

6. Hoste EAJ, Bagshaw SM, Bellomo R et al (2015) Epidemiology of acute kidney injury in critically ill patients: the multinational AKI-EPI study. Intensive Care Med 41:1411-1423. doi:10.1007/s00134-015-3934-7

7. Ostermann M, Joannidis M, Pani A et al (2016) Patient selection and timing of continuous renal replacement therapy. Blood Purif 42:224-237. doi:10.1159/000448506

8. Raimundo M, Crichton S, Martin JR et al (2015) Increased fluid administration after early acute kidney injury is associated with less renal recovery. Shock Augusta Ga 44:431-437. doi:10.1097/SHK.0000000000000453

9. Prowle JR, Liu Y-L, Licari E et al (2011) Oliguria as predictive biomarker of acute kidney injury in critically ill patients. Crit Care 15:R172. doi:10.1186/ cc10318

10. Vaara ST, Parviainen I, Pettilä V et al (2016) Association of oliguria with the development of acute kidney injury in the critically ill. Kidney Int 89:200-208. doi:10.1016/j.kint.2015.12.007

11. Prowle JR, Ishikawa K, May CN, Bellomo R (2010) Renal plasma flow and glomerular filtration rate during acute kidney injury in man. Ren Fail 32:349-355. doi:10.3109/08860221003611695

12. Calzavacca P, Evans RG, Bailey M et al (2015) Cortical and medullary tissue perfusion and oxygenation in experimental septic acute kidney injury. Crit Care Med 43:e431-e439. doi:10.1097/CCM.0000000000001198

13. Prowle JR, Molan MP, Hornsey E, Bellomo R (2012) Measurement of renal blood flow by phase-contrast magnetic resonance imaging during septic acute kidney injury: a pilot investigation. Crit Care Med 40:1768-1776. doi:10.1097/CCM.0b013e318246bd85

14. Parekh N, Veith U (1981) Renal hemodynamics and oxygen consumption during postischemic acute renal failure in the rat. Kidney Int 19:306-316

15. Redfors B, Bragadottir G, Sellgren J et al (2010) Acute renal failure is NOT an "acute renal success" - a clinical study on the renal oxygen supply/ demand relationship in acute kidney injury. Crit Care Med 38:1695-1701. doi:10.1097/CCM.0b013e3181e61911
16. Saotome T, Ishikawa K, May CN et al (2010) The impact of experimental hypoperfusion on subsequent kidney function. Intensive Care Med 36:533-540. doi:10.1007/s00134-009-1740-9

17. De Backer D, Creteur J, Preiser J-C et al (2002) Microvascular blood flow is altered in patients with sepsis. Am J Respir Crit Care Med 166:98-104

18. Lee WL, Slutsky AS (2010) Sepsis and endothelial permeability. N Engl J Med 363:689-691. doi:10.1056/NEJMcibr1007320

19. Nijssen EC, Rennenberg RJ, Nelemans PJ et al (2017) Prophylactic hydration to protect renal function from intravascular iodinated contrast material in patients at high risk of contrast-induced nephropathy (AMACING): a prospective, randomised, phase 3, controlled, open-label, non-inferiority trial. Lancet. doi:10.1016/S0140-6736(17)30057-0

20. Legrand M, Le Cam B, Perbet S et al (2016) Urine sodium concentration to predict fluid responsiveness in oliguric ICU patients: a prospective multicenter observational study. Crit Care 20:165. doi:10.1186/ s13054-016-1343-0

21. Zafrani L, Ergin B, Kapucu A, Ince C (2016) Blood transfusion improves renal oxygenation and renal function in sepsis-induced acute kidney injury in rats. Crit Care 20:406. doi:10.1186/s13054-016-1581-1

22. Hoste EA, Maitland K, Brudney CS et al (2014) Four phases of intravenous fluid therapy: a conceptual model. Br J Anaesth 113:740-747. doi:10.1093/bja/aeu300

23. Hammond NE, Taylor C, Saxena M et al (2015) Resuscitation fluid use in Australian and New Zealand intensive care units between 2007 and 2013. Intensive Care Med 41:1611-1619. doi:10.1007/s00134-015-3878-y

24. Myburgh JA, Finfer S, Bellomo R et al (2012) Hydroxyethyl starch or saline for fluid resuscitation in intensive care. N Engl J Med 367:1901-1911. doi:10.1056/NEJMoa1209759

25. Zarychanski R, Abou-Setta AM, Turgeon AF et al (2013) Association of hydroxyethyl starch administration with mortality and acute kidney injury in critically ill patients requiring volume resuscitation: a systematic review and meta-analysis. JAMA 309:678-688. doi:10.1001/jama.2013.430

26. Perner A, Haase N, Guttormsen AB et al (2012) Hydroxyethyl starch 130/0.42 versus Ringer's acetate in severe sepsis. N Engl J Med 367:124134. doi:10.1056/NEJMoa1204242

27. Haase N, Perner A, Hennings LI et al (2013) Hydroxyethyl starch 130/0.380.45 versus crystalloid or albumin in patients with sepsis: systematic review with meta-analysis and trial sequential analysis. BMJ 346:f839

28. Rochwerg B, Alhazzani W, Gibson A et al (2015) Fluid type and the use of renal replacement therapy in sepsis: a systematic review and network meta-analysis. Intensive Care Med 41:1561-1571. doi:10.1007/ s00134-015-3794-1

29. Rhodes A, Evans LE, Alhazzani W et al (2017) Surviving Sepsis Campaign: international guidelines for management of sepsis and septic shock: 2016. Intensive Care Med. doi:10.1007/s00134-017-4683-6

30. FDA (2013) FDA Safety Communication: Boxed Warning on increased mortality and severe renal injury, and additional warning on risk of bleeding, for use of hydroxyethyl starch solutions in some settings. https://www.fda.gov/ downloads/biologicsbloodvaccines/bloodbloodproducts/approvedproducts/newdrugapplicationsndas/ucm083138.pdf. Accessed 1 Jan 2017.

31. EMA (2013) Hydroxyethyl-starch solutions (HES) should no longer be used in patients with sepsis or burn injuries or in critically ill patients. http:// www.ema.europa.eu/ema/index.jsp?curl=pages/medicines/human/ referrals/Hydroxyethyl_starch-containing_solutions/human_referral_ prac 000012.jsp\&mid=WC0b01ac05805c516f. Accessed 1 Jan 2017.

32. Moeller C, Fleischmann C, Thomas-Rueddel D et al (2016) How safe is gelatin? A systematic review and meta-analysis of gelatin-containing plasma expanders vs crystalloids and albumin. J Crit Care 35:75-83. doi:10.1016/j.jcrc.2016.04.011

33. Bayer O, Reinhart K, Kohl M et al (2012) Effects of fluid resuscitation with synthetic colloids or crystalloids alone on shock reversal, fluid balance, and patient outcomes in patients with severe sepsis: a prospective sequential analysis. Crit Care Med 40:2543-2551. doi:10.1097/ CCM.0b013e318258fee7

34. Bayer O, Schwarzkopf D, Doenst T et al (2013) Perioperative fluid therapy with tetrastarch and gelatin in cardiac surgery - a prospective sequential analysis. Crit Care Med 41:2532-2542. doi:10.1097/ CCM.0b013e3182978fb6

35. Finfer S, Bellomo R, Boyce $N$ et al (2004) A comparison of albumin and saline for fluid resuscitation in the intensive care unit. N Engl J Med 350:2247-2256. doi:10.1056/NEJMoa040232 
36. Caironi P, Tognoni G, Masson S et al (2014) Albumin replacement in patients with severe sepsis or septic shock. N Engl J Med 370:1412-1421. doi:10.1056/NEJMoa1305727

37. Perel P, Roberts I, Ker K (2013) Colloids versus crystalloids for fluid resuscitation in critically ill patients. Cochrane Database Syst Rev. doi:10.1002/14651858.CD000567.pub6

38. Wilcox CS (1983) Regulation of renal blood flow by plasma chloride. J Clin Invest 71:726-735

39. Chowdhury AH, Cox EF, Francis ST, Lobo DN (2012) A randomized, controlled, double-blind crossover study on the effects of 2 - $L$ infusions of $0.9 \%$ saline and Plasma-Lyte ${ }^{\circledR} 148$ on renal blood flow velocity and renal cortical tissue perfusion in healthy volunteers. Ann Surg 256:18-24. doi:10.1097/SLA.0b013e318256be72

40. Morsing P, Velazquez H, Ellison D, Wright FS (1993) Resetting of tubuloglomerular feedback by interrupting early distal flow. Acta Physiol Scand 148:63-68. doi:10.1111/j.1748-1716.1993.tb09532.x

41. Schnermann J, Ploth DW, Hermle M (1976) Activation of tubulo-glomerular feedback by chloride transport. Pflugers Arch 362:229-240

42. Handy JM, Soni N (2008) Physiological effects of hyperchloraemia and acidosis. Br J Anaesth 101:141-150. doi:10.1093/bja/aen148

43. Morgan TJ, Venkatesh B, Hall J (2002) Crystalloid strong ion difference determines metabolic acid-base change during in vitro hemodilution. Crit Care Med 30:157-160

44. Krajewski ML, Raghunathan K, Paluszkiewicz SM et al (2015) Meta-analysis of high-versus low-chloride content in perioperative and critical care fluid resuscitation. Br J Surg 102:24-36. doi:10.1002/bjs.9651

45. Yunos NM, Bellomo R, Hegarty C et al (2012) Association between a chloride-liberal vs chloride-restrictive intravenous fluid administration strategy and kidney injury in critically ill adults. JAMA 308:1566-1572. doi:10.1001/jama.2012.13356

46. Bayer O, Reinhart K, Sakr Y et al (2011) Renal effects of synthetic colloids and crystalloids in patients with severe sepsis: a prospective sequential comparison. Crit Care Med 39:1335-1342. doi:10.1097/ cCM.0b013e318212096a

47. Yunos NM, Bellomo R, Glassford N et al (2015) Chloride-liberal vs. chloride-restrictive intravenous fluid administration and acute kidney injury: an extended analysis. Intensive Care Med 41:257-264. doi:10.1007/ s00134-014-3593-0

48. Raghunathan K, Shaw A, Nathanson B et al (2014) Association between the choice of IV crystalloid and in-hospital mortality among critically ill adults with sepsis. Crit Care Med 42:1585-1591. doi:10.1097/ CCM.0000000000000305

49. Shaw AD, Schermer CR, Lobo DN et al (2015) Impact of intravenous fluid composition on outcomes in patients with systemic inflammatory response syndrome. Crit Care 19:334. doi:10.1186/s13054-015-1045-z

50. Young $P$, Bailey $M$, Beasley R et al (2015) Effect of a buffered crystalloid solution vs saline on acute kidney injury among patients in the intensive care unit: the SPLIT randomized clinical trial. JAMA 314:1701-1710. doi:10.1001/jama.2015.12334

51. Semler MW, Wanderer JP, Ehrenfeld JM et al (2016) Balanced crystalloids versus saline in the intensive care unit: the SALT randomized trial. Am J Respir Crit Care Med. doi:10.1164/rccm.201607-1345OC

52. Sen A, Keener CM, Sileanu FE et al (2017) Chloride content of fluids used for large-volume resuscitation is associated with reduced survival. Crit Care Med 45:e146-e153. doi:10.1097/CCM.0000000000002063

53. Rivers E, Nguyen B, Havstad S et al (2001) Early goal-directed therapy in the treatment of severe sepsis and septic shock. N Engl J Med 345:13681377. doi:10.1056/NEJMoa010307

54. Pettilä V, Bellomo R (2014) Understanding acute kidney injury in sepsis. Intensive Care Med 40:1018-1020. doi:10.1007/s00134-014-3313-9

55. Chen KP, Cavender S, Lee J et al (2016) Peripheral edema, central venous pressure, and risk of AKI in critical illness. Clin J Am Soc Nephrol CJASN 11:602-608. doi:10.2215/CJN.08080715

56. Bihari S, Baldwin CE, Bersten AD (2013) Fluid balance does not predict estimated sodium balance in critically ill mechanically ventilated patients. Crit Care Resusc 15:89-96

57. Glassford NJ, Mårtensson J, Eastwood GM et al (2016) Defining the characteristics and expectations of fluid bolus therapy: a worldwide perspective. J Crit Care 35:126-132. doi:10.1016/j.jcrc.2016.05.017
58. Hjortrup PB, Haase N, Bundgaard $\mathrm{H}$ et al (2016) Restricting volumes of resuscitation fluid in adults with septic shock after initial management: the CLASSIC randomised, parallel-group, multicentre feasibility trial. Intensive Care Med 42:1695-1705. doi:10.1007/s00134-016-4500-7

59. Silversides JA, Major E, Ferguson AJ et al (2016) Conservative fluid management or deresuscitation for patients with sepsis or acute respiratory distress syndrome following the resuscitation phase of critical illness: a systematic review and meta-analysis. Intensive Care Med. doi:10.1007/ s00134-016-4573-3

60. Investigators ProCESS, Yealy DM, Kellum JA et al (2014) A randomized trial of protocol-based care for early septic shock. N Engl J Med 370:16831693. doi:10.1056/NEJMoa1401602

61. Teixeira C, Garzotto F, Piccinni P et al (2013) Fluid balance and urine volume are independent predictors of mortality in acute kidney injury. Crit Care 17:R14. doi:10.1186/cc12484

62. Vaara ST, Korhonen A-M, Kaukonen K-M et al (2012) Fluid overload is associated with an increased risk for 90-day mortality in critically ill patients with renal replacement therapy: data from the prospective FINNAKI study. Crit Care 16:R197. doi:10.1186/cc11682

63. Rosner MH, Ostermann M, Murugan R et al (2014) Indications and management of mechanical fluid removal in critical illness. Br J Anaesth 113:764-771. doi:10.1093/bja/aeu297

64. Prowle JR, Kirwan CJ, Bellomo R (2014) Fluid management for the prevention and attenuation of acute kidney injury. Nat Rev Nephrol 10:37-47. doi:10.1038/nrneph.2013.232

65. Ho KM, Sheridan DJ (2006) Meta-analysis of frusemide to prevent or treat acute renal failure. BMJ 333:420. doi:10.1136/bmj.38902.605347.7C

66. Mehta RL, Pascual MT, Soroko S et al (2002) Diuretics, mortality, and nonrecovery of renal function in acute renal failure. JAMA 288:2547-2553

67. Uchino S, Doig GS, Bellomo R et al (2004) Diuretics and mortality in acute renal failure. Crit Care Med 32:1669-1677

68. Goldstein S, Bagshaw S, Cecconi M et al (2014) Pharmacological management of fluid overload. Br J Anaesth 113:756-763. doi:10.1093/bja/aeu299

69. Schneider AG, Baldwin I, Freitag E et al (2012) Estimation of fluid status changes in critically ill patients: fluid balance chart or electronic bed weight? J Crit Care 27:745.e7-745.e12. doi:10.1016/j.jcrc.2011.12.017

70. Uszko-Lencer NHMK, Bothmer F, van Pol PEJ, Schols AMWJ (2006) Measuring body composition in chronic heart failure: a comparison of methods. Eur J Heart Fail 8:208-214. doi:10.1016/j.ejheart.2005.07.007

71. Chen H, Wu B, Gong D, Liu Z (2015) Fluid overload at start of continuous renal replacement therapy is associated with poorer clinical condition and outcome: a prospective observational study on the combined use of bioimpedance vector analysis and serum N-terminal pro-Btype natriuretic peptide measurement. Crit Care 19:135. doi:10.1186/ s13054-015-0871-3

72. Jones SL, Tanaka A, Eastwood GM et al (2015) Bioelectrical impedance vector analysis in critically ill patients: a prospective, clinician-blinded investigation. Crit Care 19:290. doi:10.1186/s13054-015-1009-3

73. Mikkelsen ME, Christie JD, Lanken PN et al (2012) The adult respiratory distress syndrome cognitive outcomes study: long-term neuropsychological function in survivors of acute lung injury. Am J Respir Crit Care Med 185:1307-1315. doi:10.1164/rccm.201111-20250C

74. Grissom CK, Hirshberg EL, Dickerson JB et al (2015) Fluid management with a simplified conservative protocol for the acute respiratory distress syndrome. Crit Care Med 43:288-295. doi:10.1097/ CCM.0000000000000715

75. Boulain T, Boisrame-Helms J, Ehrmann S et al (2015) Volume expansion in the first 4 days of shock: a prospective multicentre study in 19 French intensive care units. Intensive Care Med 41:248-256. doi:10.1007/ s00134-014-3576-1

76. Hjortrup PB, Haase N, Wetterslev J et al (2017) Effects of fluid restriction on measures of circulatory efficacy in adults with septic shock. Acta Anaesthesiol Scand. doi:10.1111/aas.12862

77. Guidet B, Martinet O, Boulain T, Philippart F, Poussel J, Maizel J, Forceville X, Feissel M, Hasselmann M, Heininger A, Aken HV (2012) Assessment of hemodynamic efficacy and safety of $6 \%$ hydroxyethylstarch 130/0.4 vs. $0.9 \% \mathrm{NaCl}$ fluid replacement in patients with severe sepsis: the CRYSTMAS study. Critical Care 16(3):R94 